\title{
Does the PillCam SB3 capsule endoscopy system improve image reading efficiency irrespective of experience? A pilot study
}

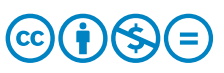

\author{
Authors \\ Nakamura ${ }^{1}$, Katsutoshi Tokushige ${ }^{1}$ \\ Institutions \\ 1 Institute of Gastroenterology, Tokyo Women's \\ University, Tokyo, Japan \\ 2 Central Clinical Laboratory, Tokyo Women's Medical \\ University, Tokyo, Japan
}

Teppei Omori ${ }^{1}$, Toshifumi Hara ${ }^{1}$, Sachiyo Sakasai ${ }^{2}$, Harutaka Kambayashi ${ }^{1}$, Shun Murasugi ${ }^{1}$, Ayumi Ito ${ }^{1}$, Shinichi

submitted 21.6.2017

accepted after revision 20.2 .2018

\author{
Bibliography \\ DOI https://doi.org/10.1055/a-0599-5852 | \\ Endoscopy International Open 2018; 06: E669-E675 \\ (c) Georg Thieme Verlag KG Stuttgart · New York \\ ISSN 2364-3722
}

Corresponding author

Teppei Omori, MD, Institute of Gastroenterology, Tokyo Women's Medical University, 8-1 Kawada-cho, Shinjuku-ku, Tokyo 162-8666, Japan

Fax: +81-3-5269-7507

tohmori@ige.twmu.ac.jp

\section{ABSTRACT}

Background and study aims The aim of this study was tp compare the diagnostic efficiency of the PillCam SB3 capsule endoscopy (CE) system with the older system, PillCam SB2, taking into consideration the experience of the image reader.

Patients and methods Small intestinal CE was conducted on 64 patients around May 2014 when the SB3 was introduced in our hospital. Data obtained from 20 patients (SB2: 10 and SB3: 10) based on transit time were assessed by junior (experience: 20 images), intermediate $(>50)$, and expert readers $(>600)$.

Results Reading time with the CE down to the end of the small intestine was shorter in the SB3 group for each reader (SB2 vs. SB3: junior, $40.2 \pm 10.1$ vs. $23.7 \pm 6.7[P=0.0009]$; intermediate, $21.4 \pm 4.9$ vs. $10.3 \pm 2.9[P=0.0003]$; expert, $23.2 \pm 5.6$ vs. $11.1 \pm 2.9 \mathrm{~min}[P=0.0002])$. Interpretation agreement rates between the findings by junior and intermediate readers and those by the expert reader were $84.6 \%$ and $92.3 \%$, respectively. For the junior reader, rates of agreement using the SB2 and SB3 systems with those by the expert reader were $85.7 \%$ and $83.3 \%$, respectively; no significant difference was noted between the two systems. Similarly, for the intermediate reader, the respective agreement rates using the SB2 and SB3 systems were $85.7 \%$ and $100 \%$, respectively.

Conclusions The PillCam SB3 reduces the time burden on readers irrespective of their experience.

\section{Introduction}

Capsule endoscopy (CE) was introduced by Iddan et al. in 2000, and PillCam (Yoqneam, Given Imaging, Atlanta, Georgia, United States) was the first capsule endoscopic system approved for clinical use in Europe and the United States in 2001. Introduction of CE has enhanced diagnosis of small intestinal diseases. CE provides noninvasive, clear visualization of the small intestine without pain [1]. With the improvement in image resolution, the viewing angle was subsequently widened from $140^{\circ}$ to $156^{\circ}$, and a second-generation PillCam SB2 with bat- tery life extension was developed. The third-generation PillCam SB3 capsule offers $30 \%$ improved image resolution compared to the PillCam SB2 and is currently in clinical use [2].

The analytical software RAPID is combined with the PillCam SB3 CE system. The latest version PillCam SB3/RAPID 8.0 or 8.3 system has improved RAPID interface, image processing functions (e.g., flexible spectral imaging color enhancement), performance (e.g., installation of a quick view mode), and improved video image reading by at least $40 \%$, compared with the PillCam SB2 system, mainly due to improvement in the software algorithm. 
- Table 1 Characteristics of participating patients and capsule endoscopy transit time to different parts of the digestive system, using the PillCam SB2 and SB3 systems

\begin{tabular}{|c|c|c|c|}
\hline \multirow[t]{2}{*}{ Parameter } & \multicolumn{2}{|l|}{ Study patients } & \multirow[t]{2}{*}{$P$ value } \\
\hline & SB2 $(n=10)$ & SB3 $(n=10)$ & \\
\hline Male/female & $5 / 5$ & $9 / 1$ & 0.1409 \\
\hline Age (mean $\pm S D$ ), years & $50 \pm 19.0$ & $48.4 \pm 20.4$ & 0.9096 \\
\hline Preparation (mosapride citrate), $\mathrm{Y} / \mathrm{N}$ & $7 / 3$ & $7 / 3$ & 1.0000 \\
\hline Esophageal transit time (sec) & $3.7 \pm 2.4$ & $2.9 \pm 1.8$ & 0.5621 \\
\hline Gastric transit time (min) & $16 \pm 17.9$ & $16 \pm 13.3$ & 0.7042 \\
\hline Small bowel transit time (min) & $244 \pm 47.3$ & $233 \pm 30.7$ & 0.6499 \\
\hline Colon sojourn time (min) & $121 \pm 52.4$ & $135 \pm 52.5$ & 0.4963 \\
\hline Total examination time (min) & $382 \pm 46.3$ & $384 \pm 43.2$ & 0.7913 \\
\hline
\end{tabular}

Video interpretation of CE is primarily the responsibility of experienced physicians, but is also sometimes performed by novice physicians, nurses who have acquired some degree of skill, and endoscopic technicians. However, it is not clear whether the third-generation CE system provides efficient video image reading in all cases.

The aim of the current study was to verify whether the thirdgeneration PillCam SB3/RAPID 8.3CE system allows reliable video image reading by readers with variable degrees of experience.

\section{Patients and methods}

A series of 64 patients underwent small intestinal CE around May 2014, when the PillCam SB3 system was introduced at our hospital. Patients enrolled in this retrospective study were divided into two groups: the PillCam SB2 system group (SB2 group; $n=30$, including 15 with obscure gastrointestinal bleeding (OGIB), 9 with Crohn's disease, and 6 with miscellaneous diseases; 18 men/12 women; age: $52.8 \pm 19.3$ years; mean small bowel transit time: $262 \pm 170 \mathrm{~min}$ [range, $40-857 \mathrm{~min}$ ], mean \pm SD), and PillCam SB3 system group (SB3 group; $n=34$, including 10 with OGIB, 10 with Crohn's disease, and 14 with miscellaneous gastrointestinal diseases; 25 men/9 women; age: $49.1 \pm 20.2$ years; small bowel transit time: $233 \pm 134 \mathrm{~min}$ [76-636 min]). This study was conducted on a series of $64 \mathrm{pa}-$ tients. The small bowel transit time varied widely among the patients. Therefore, to reduce the effect of this variable on the results, 10 patients from each group with small intestine transit times closest to the mean value for that group were selected for inclusion in this study ( $\triangleright$ Table 1 ). Mean transit times for the analyzed groups were $244 \pm 47$ minutes in the SB2 group and $233 \pm 31$ minutes in the SB3 group. First, we obtained clinical data on the 20 selected patients from their medical records. Second, the cases were anonymized, and the data randomized. Third, three readers with different degrees of experience, who were blinded to the clinical data, were asked to read the obtained CE images from the 20 patients ( $\downarrow$ Table 2 ). The images were interpreted after preparation of thumbnails of the esoph- agus, stomach, duodenum, and cecum. During examination, each reader created thumbnails and commented on each image. Video image reading time was defined as the time required for playback of all images from start to end of video and creation of all thumbnails. Image reading times of the SB2 and SB3 groups and number of thumbnails of significant findings created by each reader were compared. The rate of diagnosis of significant findings among the readers was also compared. Patients who did not present with significant findings were also included in this study.

\section{Image reading setting}

CE was conducted in the standard manner. Routine medications were administered as usual, and the patients were instructed to fast for more than 12 hours from 21:00 the day before examination. Dimethicone $(15 \mathrm{~mL})$ was administered orally 30 minutes before the examination. The CE (with Pillcam SB2 or SB3) was swallowed with tap water. Two hours after the start of examination, drinking water was allowed and a meal was provided 4 hours after swallowing the CE. After completion of the examination, excretion of the capsule was confirmed visually.

The PillCam SB2 system consists of a DR2 recorder, PillCam SB2 capsule endoscopy, and RAPID ver. 6.5 video production software (Given Imaging, Yokneam Illit, Israel). The PillCam SB3 system consists of a DR3 recorder, PillCam SB3 capsule endoscopy, and RAPID ver. 8.0 video creation software. The RAPID ver. 8.0 software was used for interpretation of all recordings (including those by PillCam SB2). After creating the starting thumbnail of the digestive tract, image reading was performed. The method of image reading was as follows: With the PillCam SB2 system, the recorded data were stored in manual mode using four screens at 28 times speed ( 1 screen/7 times speed), while in the PillCam SB3 system, the recorded data were stored in the review mode using four screens at 28 times speed ( 1 screen/7 times speed). 
Table 2 Purpose of capsule endoscopy examination and main findings in 20 patients.

\begin{tabular}{|c|c|c|c|}
\hline Patient no. & SB & Purpose of examination & Main capsule endoscopy findings \\
\hline 1 & SB2 & OGIB & Angioectasia \\
\hline 2 & SB2 & Crohn's disease & Erosion/ulceration \\
\hline 3 & SB2 & Suspected ischemic enteritis & No significant findings (lymphangiectasia) \\
\hline 4 & SB3 & Cronkhite-Canada syndrome & Cronkhite-Canada polyps \\
\hline 5 & SB3 & Crohn's disease & Erosion \\
\hline 6 & SB2 & Crohn's disease & Erosion/ulceration \\
\hline 7 & SB3 & Crohn's disease & Erosion \\
\hline 8 & SB2 & Cronkhite-Canada syndrome & Cronkhite-Canada polyps \\
\hline 9 & SB2 & Suspected tumor & No significant findings (red spot) \\
\hline 10 & SB2 & OGIB & Polyp \\
\hline 11 & SB3 & OGIB & Diverticulum \\
\hline 12 & SB3 & Suspected Crohn's disease & No significant findings (red spot, lymphangiectasia) \\
\hline 13 & SB3 & OGIB & No significant findings (red spot, lymphangiectasia) \\
\hline 14 & SB3 & Bechet disease & No significant findings (red spot) \\
\hline 15 & SB3 & Ulcerative colitis & No significant findings (red spot) \\
\hline 16 & SB2 & OGIB & No significant findings \\
\hline 17 & SB3 & Crohn's disease & Erosion/ulceration \\
\hline 18 & SB3 & Crohn's disease & Erosion/ulceration \\
\hline 19 & SB2 & OGIB & Diverticulum \\
\hline 20 & SB2 & Crohn's disease & Angioectasia \\
\hline
\end{tabular}

\section{Definition of readers}

Readers (T.O., T.H., and S.S.) with different experience levels interpreted the recorded data. The junior reader (S. S.) completed the image reading training program formulated by the Japanese Association for Capsule Endoscopy and was a clinical laboratory technician with past experience of 20 video image readings. The intermediate reader was a gastroenterologist (T.H.) who had completed more than 50 video image readings. The expert reader (T. O.) was a gastroenterological endoscopist who had performed more than 600 video image readings.

\section{Ethical considerations}

All patients fulfilled the eligibility criteria for $C E$. The need for CE examination, its risks, and countermeasures against potential complications were carefully explained to each patient, and written informed consent was obtained. The study protocol was reviewed and approved by the Human Ethics Review Committee of our university [registry number 4115].

\section{Statistical analysis}

All data are expressed as mean \pm standard deviation. Differences between groups were analyzed with Fischer's exact test and $t$-test or Wilcoxon's rank test, as appropriate. A $P$ value $<0.05$ indicated statistically significant difference. JMP statistical analysis software (version 11, SAS, Cary, NC) was used in all analyses.

\section{Results}

There were no significant differences in clinical background of the SB2 and SB3 patients, including age, sex distribution, and rate of background gastrointestinal diseases. Gastrointestinal transit times were similar between the two groups, including esophageal, gastric, small intestinal, and large intestinal transit times, as well as total transit time. In all cases, CE completed imaging of the small intestine within the examination time, and we were able to examine the small intestine ( $\triangleright$ Table 1 ). Image reading times from the start of reading to the end of the small intestine were as follows for the SB2 and SB3 groups: junior: $40.2 \pm 10.1$ vs. $23.7 \pm 6.7$ minutes $(P=0.0009)$, intermediate: $21.4 \pm 4.9$ vs. $10.3 \pm 2.9 \mathrm{~min}(P=0.0003)$, and expert: 


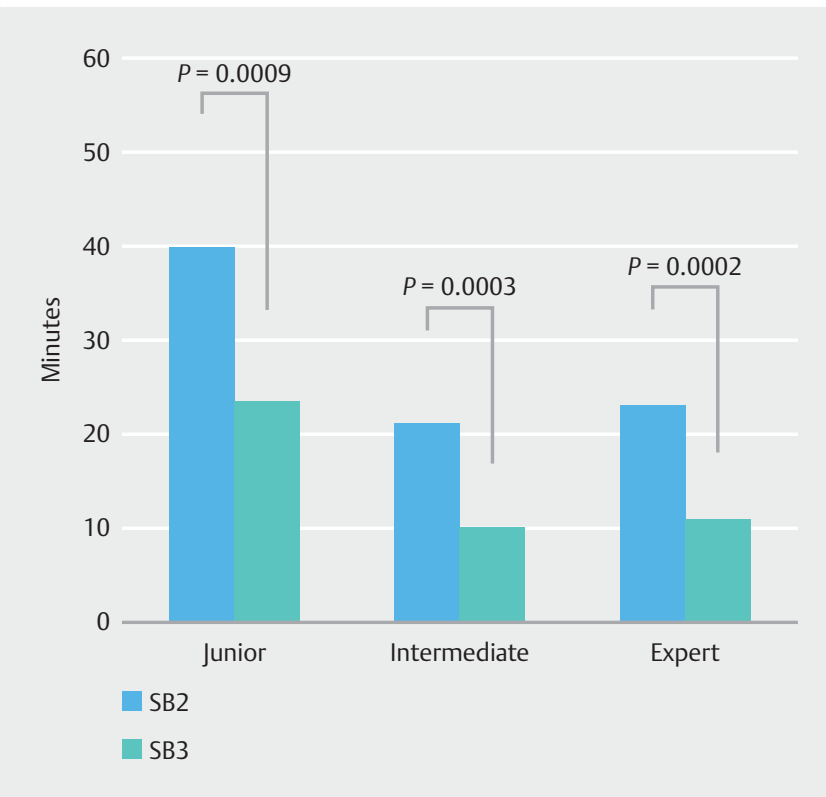

- Fig. 1 Reading time with the capsule advanced down to the end of the small intestine, according to reader experience and generation of the system used. Data are mean \pm SD. $P$ values determined by Student's $t$-test.

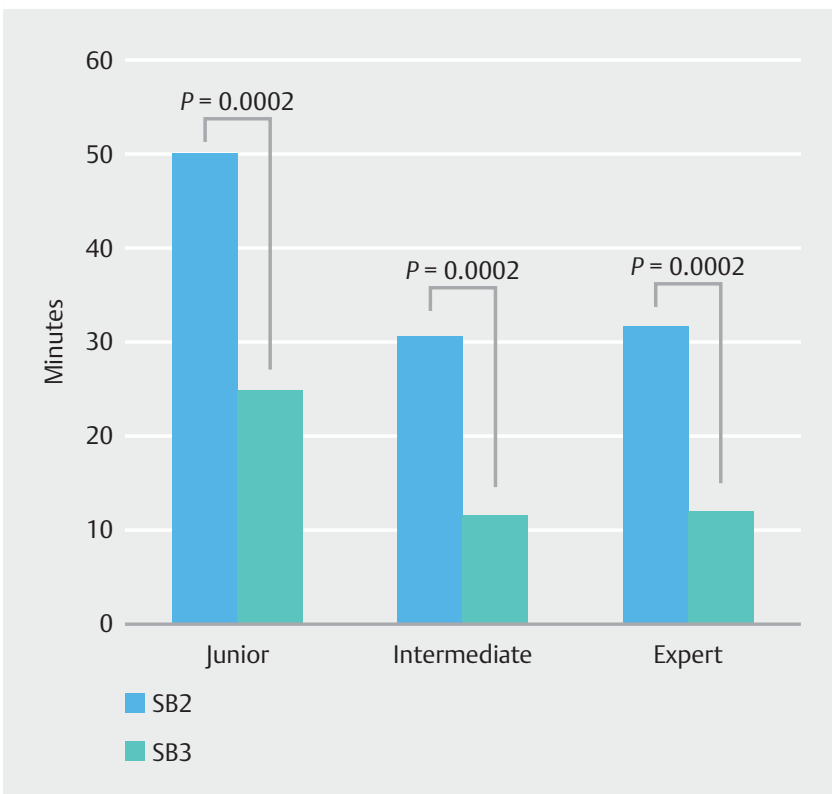

- Fig. 2 Total reading time with the capsule advanced down to the end of the large intestine, according to reader experience and generation of the system used. Data are mean \pm SD. $P$ values determined by Student's $t$-test.

$23.2 \pm 5.6$ vs. $11.1 \pm 2.9 \min (P=0.0002)$. As shown in > Fig. 1 , the SB3 system shortened reading time for all readers.

Reading times for the entire digestive system, including the colon, were as follows for the SB2 and SB3 groups: junior: $49.9 \pm$ 10.4 vs. $24.9 \pm 6.7(P=0.0002)$, intermediate: $30.4 \pm 6.4$ vs. $11.4 \pm 3.7(P=0.0002)$, and expert: $31.7 \pm 4.1$ vs. $12.1 \pm 3.4$ min-

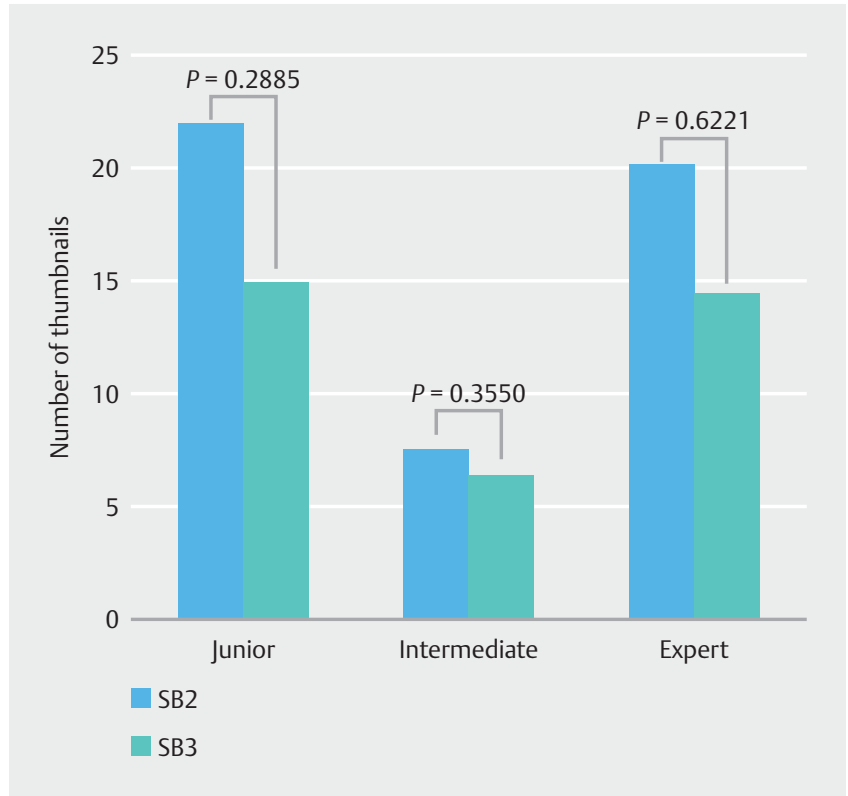

Fig. 3 Number of thumbnails created by the reader, according to experience and generation of the system used. Data are mean \pm SD. $P$ values determined by Student's $t$-test.

utes $(P=0.0002)$. Similarly, reading time correlated significantly with the SB3 group ( $\triangleright$ Fig. 2). The number of created thumbnails was as follows for the SB2 and SB3 groups: junior: $22.1 \pm$ 14.3 vs. $15.0 \pm 9.49(P=0.2885)$, intermediate: $7.5 \pm 3.2$ vs. $6.5 \pm 3.1 \quad(P=0.3550)$, expert: $20.2 \pm 14.7$ vs. $14.5 \pm 5.6(P=$ $0.6221)$. There was no significant difference between the two versions for any reader (> Fig. $\mathbf{3}$ ).

Significant findings were identified in 13 of 20 patients (65\%; > Table2). When the data from the expert reader were used as the reference, there was a high agreement rate for detection of main findings in patients who presented with significant clinical findings. Readings from the junior and intermediate readers agreed significantly with those of the expert reader at rates of $84.6 \%$ and $92.3 \%$, respectively, using data from the SB2 and SB3 systems. Using data from the SB2 system alone, rates of agreement between readings from the junior and intermediate readers and those of the expert reader were $85.7 \%$ and $85.7 \%$, respectively, while the respective agreement rates for data from the SB3 system alone were $83.3 \%$ and $100 \%$. There were no significant differences in agreement rates between SB2 and SB3 for either the junior or intermediate reader (> Fig.4).

\section{Discussion}

It was previously reported that interpretation of CE imaging requires a considerable amount of time, placing a significant burden on the reader $[3,4]$. There are also reports that described reading of CE images by health professionals other than gastroenterologists/endoscopists, e.g., nurses [5-9]. In this regard, improvements in the CE system should reduce the burden on the reader, with improvement in reading efficiency based on 
Junior



Intermediate



- Fig. 4 Agreement rates for interpretation made by the junior and intermediate readers relative to that of the expert reader, using the SB2 and SB3 systems. Data are mean \pm SD. $P$ values determined by Student's $t$-test.

improvements in the software algorithm [3,9-12]. The thirdgeneration CE system (PillCam SB3) is used in combination with RAPID software (version 8.0 or 8.3 ). Compared with the PillCam SB2 system, the PillCam SB3 is considered to optimize video interpretation by $\geq 40 \%$ based on the new algorithm [2]. With the PillCam SB3, image reading is sometimes handled by junior physicians, nurses who have acquired a certain degree of skill, and endoscopic technicians, in addition to experienced physicians. Before wider adoption of this system, it is important to clarify whether efficient video image reading could be provided by readers other than expert endoscopists.

In the current study, we compared image interpretation by three readers with differing levels of clinical expertise and experience with the CE system generation (second- and thirdgeneration systems); the latter was represented by the number of video images interpreted by the reader. Use of the third-generation system clearly shortened interpretation time not only by the experts but also by inexperienced readers.

The reading mode for the second-generation CE system includes manual and automatic modes, and the manual mode was used in the current study for interpretation of all recorded images. In the third-generation system, the manual and automatic modes have been replaced by the review mode. The PillCam SB3 system is equipped with a function that can increase the adaptive frame rate (number of images captured per second when the capsule moves quickly inside the intestinal tract) from two to six pictures. It is a concern that the larger the frame rate, the more time is needed for interpretation. Therefore, in the video recording stage of the PillCam SB3 system, captured images that are very similar are deleted, making it possible to create simple video data. In the current study, we compared interpretation time with the PillCam SB2 and PillCam SB3 systems, on the premise that all created video data were interpreted. Small-bowel CE is mainly for evaluation of the small intestine. Therefore, it was necessary to compare the small intestine interpretation time. However, in small bowel capsule endoscopic examination, images of the colon from arrival at the colon to the end of the examination are also captured. When performing small-bowel CE, pathological features may be found in the colon, even though this segment of the intestine was not prepared [13]. Therefore, it is also important to check the captured images of the colon during small bowel CE. The improved algorithm has the potential to significantly reduce the number of images corresponding to normal colon findings. In this study, both interpretation time to the end of the small intestine and interpretation time up to the end of the examination, including the colon, were compared for each reader. The results showed that not only a reduction in colorectal data but also improvement in efficiency of interpretation of the small intestine are responsible for shortening the interpretation time using the new-generation system. It is conceivable that the improved software algorithm contributed to the reduction in reading time. Furthermore, the improved capsule endoscope itself improved image resolution and image reading; using better images becomes possible, although the viewing angle remains unchanged.

There are only a few publications on the PillCam SB3 CE system. Monteiro et al. [14] compared rates of detection rates of 
duodenal papilla with the SB2 and SB3 systems and reported a significantly higher duodenal papilla detection rate using the SB3 system, indicating that this system offers a better likelihood of accurately diagnosing pathologies in the proximal part of the small intestine. In addition, Kunihara et al. [15] reported that the SB3 system is superior to the SB2 system in detection of esophageal varices. To our knowledge, however, no reports have compared the image reading time between the two systems. Also, the current study is the first to report the effects of reader experience on image interpretation between the two systems.

The role played by other health professionals "pre-readers," such as nurses, in interpretation of CE has been discussed in Europe and the United States $[6,7,11,16]$. It has been reported that non-physician assistants can contribute toward shortening the reading time and improving the diagnostic ability of physicians [6, 7]. Therefore, new approaches, such as examination of the learning curve, training, and certification system, have been reported $[11,16]$. Reducing the burden of image reading is necessary not only for physicians skilled in CE image reading, but also for relatively inexperienced physicians as well as nurses and clinical laboratory technologists who act as pre-readers. In the current study, a clinical laboratory technician participated as the junior reader. Rates of significant findings on analysis of the $\mathrm{CE}$ systems by the junior and intermediate readers were $84.6 \%$ and $92.3 \%$, respectively, compared with findings by the skilled endoscopist. Moreover, for the junior reader, the aforementioned rates using the SB2 and SB3 systems were $85.7 \%$ and $83.3 \%$, respectively, compared with experts, and there was no significant difference between the two systems. Similarly, for the intermediate reader, the aforementioned rates using the SB2 and SB3 systems were $85.7 \%$ and $100 \%$, respectively, compared with the experts. There was no significant difference between the two systems. Based on these findings, we conclude that accurate interpretation of the recorded data is not affected by experience when using the new system. In addition, the junior reader not only lacked experience but also differed from the other two readers in that she was not a physician. Interpretation using this new system may reduce the burden on the pre-readers.

This study has several limitations. First, the sample size was small. Second, our study included some cases that lacked characteristic findings in order to avoid selection bias. The current study compared results from three readers with different levels of image reading experience. Regarding the sample size, the SB2 and SB3 videos were restricted to 10 patients each for experienced readers. If the sample size were larger, the reading skill of a non-experienced reader might improve with time during the study given the additional reading experience [11]. In addition, the intermediate reader checked fewer thumbnails than the other readers. Junior readers tend to read more thumbnails, regardless of significant findings [8]. Intermediate readers are becoming more accustomed to interpretation. Therefore, we think that intermediate readers may reduce the number of thumbnails of lesions to reduce the burden of image interpretation. However, as shown in this study, significant findings that contributed to diagnosis were captured by both junior and intermediate readers.

\section{Conclusion}

In conclusion, the third-generation CE system with RAPID 8.0 or 8.3 software, PillCam SB3, and DR3 recorder makes it easier to accurately interpret recorded images irrespective of image reading experience and shortened reading time, compared with the SB2 system. We conclude that examination using the PillCam SB3 system can reduce the burden on the reader.

\section{Acknowledgements}

The authors thank Dr. S. Shimizu for advice on statistical analysis. We also thank Word-Medex (http://www.word-medex.com. au) for reading and editing the manuscript.

\section{Competing interests}

None

References

[1] Iddan G, Meron G, Glukhovsky A et al. Wireless capsule endoscopy. Nature 2000; 405: 417

[2] PillCam SB. Medtronic. Available at: 2017: http://www.medtronic. com/covidien/products/capsule-endoscopy/pillcam-sb-3-system [Accessed March 10, 2017]

[3] Kyriakos N, Karagiannis S, Galanis P et al. Evaluation of four time-saving methods of reading capsule endoscopy videos. Eur J Gastroenterol Hepatol 2012; 24: 1276-1280

[4] Barkin JA, Barkin JS. Video capsule endoscopy: Technology, reading, and troubleshooting. Gastrointest Endoscopy Clin N Am 2017; 27: $15-27$

[5] Drew K, McAlindon ME, Sanders DS et al. The nurse endoscopist: moving ahead with time. Gastroenterol Nurs 2013; 36: 209-213

[6] Brock AS, Freeman J, Roberts J et al. A resource-efficient tool for training novices in wireless capsule endoscopy. Gastroenterol Nurs 2012; 35: 317-321

[7] Shiotani A, Honda K, Kawakami M et al. Analysis of small-bowel capsule endoscopy reading by using Quickview mode: training assistants for reading may produce a high diagnostic yield and save time for physicians. J Clin Gastroenterol 2012; 46: e92-95

[8] Niv Y, Niv G. Capsule endoscopy examination-preliminary review by a nurse. Dig Dis Sci 2005; 50: 2121 - 2124

[9] Shiotani A, Honda K, Kawakami M et al. Evaluation of RAPID $\left({ }^{\circledR}\right) 5$ Access software for examination of capsule endoscopies and reading of the capsule by an endoscopy nurse. J Gastroenterol 2011; 46: 138 142

[10] Saurin JC, Lapalus MG, Cholet F et al. French Society of Digestive Endoscopy (SFED). Can we shorten the small-bowel capsule reading time with the "Quick-view" image detection system? Dig Liver Dis 2012; 44: 477-481

[11] Hosoe N, Rey JF, Imaeda H et al. Evaluations of capsule endoscopy software in reducing the reading time and the rate of false negatives by inexperienced endoscopists. Clin Res Hepatol Gastroenterol 2012; 36: $66-71$ 
[12] Günther U, Daum S, Zeitz M et al. Capsule endoscopy: comparison of two different reading modes. Int J Colorectal Dis 2012; 27: 521 - 525

[13] Juanmartiñena Fernández JF, Fernández-Urién Sainz I, Zabalza Ollo B et al. Colonic lesions in patients undergoing small bowel capsule endoscopy: incidence, diagnostic and therapeutic impact. Rev Esp Enferm Dig 2017; 109: 498-502
[14] Monteiro S, de Castro FD, Carvalho PB et al. PillCam ${ }^{\circledR}$ SB3 capsule: Does the increased frame rate eliminate the risk of missing lesions? World J Gastroenterol 2016; 22: 3066-3068

[15] Kunihara S, Oka S, Tanaka S et al. Third-Generation Capsule Endoscopy Outperforms Second-Generation Based on the Detectability of Esophageal Varices. Gastroenterol Res Pract 2016; 2016: 9671327

[16] McAlindon ME, Ching HL, Yung D et al. Capsule endoscopy of the small bowel. Ann Transl Med 2016; 4: 369 